ESAIM: Proceedings, Vol. 4, 1998, 117-135

Contrôle et Équations Aux DÉRivées Partielles

http://www.emath.fr/proc/Vol.4/

\title{
MODELING AND ANALYSIS OF MULTILAYER LAMINATED PLATES
}

\author{
SCOTT W. HANSEN \\ Department of Mathematics \\ Iowa State University \\ Ames, Iowa 50011 USA
}

Key Words : laminated plate, multilayer plate, constrained layer plate.

AMS Subject Qualification : 73K35, 73K50, 73B25, 73D30.

Article published by EDP Sciences and available at http://www.edpsciences.org/proc or http://dx.doi.org/10.1051/proc:1998024 


\begin{abstract}
A dynamic model for a multilayered laminated plate is developed. The laminated plate consists of $2 n$ plate layers and $2 n-1$ adhesive layers. The layers (both plate and adhesive layers) are assumed to be homogeneous, transversely isotropic and perfectly bonded to one another. In the initial modeling, the Reissner-Mindlin theory of shear deformable plates is applied to each layer, resulting in a high-order plate theory in which the shear motions of the layers are completely independent. Simpler, lowerorder models are obtained from the initial model from asymptotic limits based upon the assumptions that (i) the adhesive layers are very thin, (ii) the elastic modulii of the adhesive layers are small compared to those of the plate layers, (iii) the shear stiffnesses of the plate layers are very large.
\end{abstract}




\section{Introduction}

Since the pioneering work on shear deformable beams and plates of Reissner [5] and later Mindlin [4], numerous models have been developed to predict the dynamic behavior of thin multilayered plates. In particular many of the constrained layer models (see Sun and $\mathrm{Lu}$ [6] for a survey and many references) have been valuable in analyzing the dynamical properties of these plates. These constrained-layer models are typically derived by assuming within each layer the assumptions used in the Mindlin or Reissner theory (namely that displacements vary linearly through the thickness, with the transverse displacements being constant with respect to the transverse direction) and then making various ad-hoc assumptions that couple the motions of each of the layers with one another. For example, in the theory of Yan and Dowell [7], the transverse shear strains are set equal at the interfaces, while in the Mead-Markus model [3] the transverse shear stresses are equated at the interfaces. Although these sound like natural assumptions, when either of these is coupled with continuity of displacement across the interfaces, one is left with two conditions at each interface. This effectively forces a direct coupling between the motion of all the layers in the sense that a motion in one layer uniquely determines the motion in all other layers.

Thus, unless one knows the extent to which these ad-hoc assumptions are valid, it is difficult to make any assesments concerning the validity the model. The approach described here partly overcomes this problem by starting with a high-order plate model in which no interface conditions, other than continuity of displacement, are assumed. By applying physically natural asymptotic assumptions, this initial high-order model can be used to obtain lower-order models with limited degrees of freedom. One then has a basis of comparison with which to assess the validity of the lower-order models.

In this article we describe how this approach applies to the modeling of a multilayer laminated material. However the same idea is valid for constrainedlayer models, modeling of plates with piezoelectric patches, combinations of each (called active constrained layer dampers) and other similar situations. For simplicity we will only consider the undamped free motions in this article, although adjustments can easily be made to include damping and external forces.

We apply in our initial modeling a multilayer version [1] of the ReissnerMindlin theory of shear deformable plates. This is a high-order plate theory in which the shear motions of each layer are completely independent. Simpler, lower-order models are then be obtained from this initial model from asymptotic limits based upon the assumptions that (i) the adhesive layers are very thin, (ii)

ESAIM: Proc., Vol. 4, 1998, 117-135 
the elastic modulii of the adhesive layers are small compared to those of the plate layers, (iii) the shear stiffnesses of the plate layers are very large. Additional simplification, that is relevant to low-frequency motions, is obtained by assuming certain rotational inertia terms are negligible. The resulting laminated plate model is a generalization of the model described in Hansen [2], in which only one adhesive layer is present.

Our general approach is valid for general (nonsymmetric) laminated plates, however for simplicity of exposition we consider in this article the symmetric case in which all the material parameters are symmetric with respect to the geometric midsheet of the plate.

This article is organized as follows. In Section 2 we review the basic assumptions used in the derivation of the multilayer plate model of Hansen [1]. In Section 3 we obtain the lower order models by taking asymptotic limits of the initial model. Some asymptotic and spectral properties of these resulting models are investigated, such as the dependence of the plate stiffness upon the glue stiffness and frequency of vibration.

\section{Multilayer plates}

The laminated plate models we will consider are all based upon the multilayer plate model in Hansen [1]. This multilayer plate model is derived under the assumptions that no slip occurs along the interfaces and each of the layers satisfy the assumptions of Reissner-Mindlin plate theory [5, 4], namely, within each layer the in-plane displacements vary linearly with respect to the transverse coordinate and the transverse displacements are constant with respect to the transverse coordinate.

Although we are mainly interested in the case of a symmetric multilayer plate, our approach is valid for nonsymmetric plates. In the following subsection we briefly describe the general $n$-layer model in [1] before specializing to the symmetric case.

\subsection{General $n$-layer plate model}

The plate is assumed to consist of $n$ plate layers which occupy the region $\Omega \times$ $(-h / 2, h / 2)$ at equilibrium, where $\Omega$ is a bounded domain in $\mathbf{R}^{2}$ with boundary $\Gamma$. Let

$$
-h / 2=z_{0}<z_{1}<\ldots<z_{n-1}<z_{n}=h / 2, \quad h_{i}=z_{i}-z_{i-1}, i=1,2, \ldots, n
$$


We use the rectangular coordinates $\underline{x}=\left\{x_{1}, x_{2}\right\}$ to denote points in $\Omega$ and $x=\left\{\underline{x}, x_{3}\right\}=\left\{x_{1}, x_{2}, x_{3}\right\}$ to denote points in $Q=\cup_{i=1}^{n} Q_{i}$, where

$$
Q_{i}=\Omega \times\left(z_{i-1}, z_{i}\right), \quad i=1,2, \ldots, n .
$$

For $x \in Q$ let $U(x)=\left\{U_{1}, U_{2}, U_{3}\right\}(x)$ denote the displacement vector of the point which, when the plate in equilibrium has coordinates $x=\left\{x_{1}, x_{2}, x_{3}\right\}$. In addition define $u^{i}=\left\{u_{1}^{i}, u_{2}^{i}\right\}$ and $u_{3}^{i}, i=0,1,2, \ldots n$ by

$$
u_{j}^{i}(\underline{x})=U_{j}\left(\underline{x}, z_{i}\right) \quad j=1,2,3, \quad \forall \underline{x} \in \Omega .
$$

Throughout this paper the index $i$ will refer to a particular layer or interface within the composite plate. For vector quantities whose components vary from layer to layer, the index $i$ will be superscripted, while for scalar quantities the $i$ will be subscripted.

Let $\sigma_{j k}, \epsilon_{j k}(j, k=1,2,3)$ denote the stress and strain tensors, respectively. For a small displacement theory we assume

$$
\epsilon_{j k}(x)=\frac{1}{2}\left(\frac{\partial U_{j}(x)}{\partial x_{k}}+\frac{\partial U_{k}(x)}{\partial x_{j}}\right), \quad \forall x \in Q .
$$

Each layer is assumed to be homogeneous and transversely isotropic, however the material properties can vary from layer to layer. Following Mindlin's approach [4], the constitutive equations for 3-dimensional transversely isotropic elasticity are reduced to following constitutive equations by assuming $\sigma_{33}$ to be negligible.

$$
\begin{array}{ll}
\sigma_{11}=\frac{E_{i}}{1-\nu_{i}^{2}}\left(\epsilon_{11}+\nu_{i} \epsilon_{22}\right) & \sigma_{12}=\frac{E_{i}}{1+\nu_{i}} \epsilon_{12} \\
\sigma_{22}=\frac{E_{i}}{1-\nu_{i}^{2}}\left(\nu_{i} \epsilon_{11}+\epsilon_{22}\right) & \sigma_{13}=2 G_{i} \epsilon_{13} \\
\sigma_{33}=0 & \sigma_{23}=2 G_{i} \epsilon_{23},
\end{array}
$$

where $E_{i}$ denotes the in-plane Young's modulus, $G_{i}$ denotes the transverse shear modulus (with shear correction [4] included), and $\nu_{i}$ denotes the in-plane Poisson's ratio, all for the $i$ th layer.

Due to the assumption that the transverse displacements are constant in the transverse direction, we may simply define the scalar function $w$ as the transverse displacement. Thus

$$
w(\underline{x}) \equiv u_{3}^{0}(\underline{x})=u_{3}^{1}(\underline{x})=\ldots=u_{3}^{n}(\underline{x}) \quad \forall \underline{x} \in \Omega .
$$

Due to the assumption that no slip occurs along the ineterfaces we find that each filament that is originally orthogonal to the surfaces has $2 n+3$ degrees of freedom: $w, u^{0}, \ldots, u^{n}$.

ESAIM: Proc., Vol. 4, 1998, 117-135 
For $i=1,2, \ldots, n$ define $\psi^{i}=\left\{\psi_{1}^{i}, \psi_{2}^{i}\right\}, \varphi^{i}=\left\{\varphi_{1}^{i}, \varphi_{2}^{i}\right\}$, and $v^{i}=\left\{v_{1}^{i}, v_{2}^{1}\right\}$ by

$$
\psi^{i}=\frac{u^{i}-u^{i-1}}{h_{i}}, \quad \varphi^{i}=\psi^{i}+(\nabla w)^{T}, \quad v^{i}=\frac{u^{i-1}+u^{i}}{2} .
$$

In addition, let $u, \psi, v, \varphi$ denote the matrices whose $i$ th row is $u^{i}, \psi^{i}, v^{i}, \varphi^{i}$, respectively. Furthermore let $\vec{\nabla} w$ denote the $n \times 2$ matrix whose $i$ th row is $(\nabla w)^{T}$. The components $\psi_{j}^{i}$ of $\psi^{i}$ can be viewed as the total rotation angles of the deformed filament within the $i$-th layer in the $x_{j}-x_{3}$ plane (with negative orientation). The components of $\varphi$ represent the (small angle approximation for the) shear angles within each layer. The components of $v^{i}$ represent the in-plane displacements of the midplanes of the $i$-th layer.

Define $\hat{z}_{i}=\left(z_{i-1}+z_{i}\right) / 2$. The displacements within the $i$ th layer can be written as

$$
\begin{array}{rlr}
U_{1}\left(\underline{x}, x_{3}\right) & =v_{1}^{i}(\underline{x})+\left(x_{3}-\hat{z}_{i}\right) \psi_{1}^{i}(\underline{x}) & z_{i-1}<x_{3}<z_{i} \\
U_{2}\left(\underline{x}, x_{3}\right) & =v_{2}^{i}(\underline{x})+\left(x_{3}-\hat{z}_{i}\right) \psi_{2}^{i}(\underline{x}) & z_{i-1}<x_{3}<z_{i} \\
U_{3}\left(\underline{x}, x_{3}\right) & =w(\underline{x}) & z_{i-1}<x_{3}<z_{i} .
\end{array}
$$

The displacement equations (4) should be interpreted in terms of the state variables $\{u, w\}$; that is, $v$ and $\psi$ are actually functions of the state $\{u, w\}$ by (3). In particular, continuity of displacements along the interfaces is automatic.

Substituting (4) into (1) gives an expression for the strain within the $i$-th layer:

$$
\begin{aligned}
\epsilon_{11} & =\frac{\partial v_{1}^{i}}{\partial x_{1}}+\left(x_{3}-\hat{z}_{i}\right) \frac{\partial \psi_{1}^{i}}{\partial x_{1}} \quad \epsilon_{22}=\frac{\partial v_{2}^{i}}{\partial x_{2}}+\left(x_{3}-\hat{z}_{i}\right) \frac{\partial \psi_{2}^{i}}{\partial x_{2}} \\
\epsilon_{12} & =\frac{1}{2}\left[\frac{\partial v_{1}^{i}}{\partial x_{2}}+\frac{\partial v_{2}^{i}}{\partial x_{1}}+\left(x_{3}-\hat{z}_{i}\right)\left(\frac{\partial \psi_{1}^{i}}{\partial x_{2}}+\frac{\partial \psi_{2}^{i}}{\partial x_{1}}\right)\right] \\
\epsilon_{13} & =\frac{1}{2}\left(\varphi_{1}^{i}\right) \quad \epsilon_{23}=\frac{1}{2}\left(\varphi_{2}^{i}\right) .
\end{aligned}
$$

Since $\sigma_{33}$ is assumed to be negligible we may assume (for the purpose of calculating the energy) that $\epsilon_{33}=0$.

The strain energy $\mathcal{P}=\sum_{i=1}^{n} \mathcal{P}_{i}$ and kinetic energy $\mathcal{K}=\sum_{i=1}^{n} \mathcal{K}_{i}$ for the composite plate are given by

$$
\mathcal{P}_{i}=\frac{1}{2} \int_{Q_{i}} \sum_{j, k=1}^{3} \epsilon_{j k} \sigma_{j k} d \underline{x} d x_{3}, \quad \mathcal{K}_{i}=\frac{1}{2} \int_{Q_{i}} \rho_{i}\left(\dot{U}_{1}^{2}+\dot{U}_{2}^{2}+\dot{U}_{3}^{2}\right) d \underline{x} d x_{3}
$$

where $=d / d t$ and $\rho_{i}>0$ denotes the mass density per unit volume within the $i$-th layer. ¿From (2) and (5) the strain energy of the $i$ th layer can be written 


$$
\begin{gathered}
\mathcal{P}_{i}=\frac{h_{i}^{3}}{2} \int_{\Omega} D_{i}\left[\left(\frac{\partial \psi_{1}^{i}}{\partial x_{1}}\right)^{2}+\left(\frac{\partial \psi_{2}^{i}}{\partial x_{2}}\right)^{2}+2 \nu_{i}\left(\frac{\partial \psi_{2}^{i}}{\partial x_{2}} \frac{\partial \psi_{1}^{i}}{\partial x_{1}}\right)+\left(\frac{1-\nu_{i}}{2}\right)\left(\frac{\partial \psi_{1}^{i}}{\partial x_{2}}+\frac{\partial \psi_{2}^{i}}{\partial x_{1}}\right)^{2}\right] d \underline{x} \\
+\frac{h_{i}}{2} \int_{\Omega} 12 D_{i}\left[\left(\frac{\partial v_{1}^{i}}{\partial x_{1}}\right)^{2}+\left(\frac{\partial v_{2}^{i}}{\partial x_{2}}\right)^{2}+2 \nu_{i}\left(\frac{\partial v_{1}^{i}}{\partial x_{1}} \frac{\partial v_{2}^{i}}{\partial x_{2}}\right)\right. \\
\left.+\left(\frac{1-\nu_{i}}{2}\right)\left(\frac{\partial v_{1}^{i}}{\partial x_{2}}+\frac{\partial v_{2}^{i}}{\partial x_{1}}\right)^{2}\right]+G_{i}\left(\left(\varphi_{1}^{i}\right)^{2}+\left(\varphi_{2}^{i}\right)^{2}\right) d \underline{x}
\end{gathered}
$$

where $D_{i}=E_{i} / 12\left(1-\nu_{i}^{2}\right) . D_{i} h_{i}^{3}$ is the modulus of flexural rigidity for the $i$ th layer and $h_{i} G_{i}$ is the modulus of elasticity in shear for the $i$ th layer. Likewise the kinetic energy of the $i$ th layer is

$$
\mathcal{K}_{i}=\frac{1}{2} \int_{\Omega} \rho_{i} h_{i}(\dot{w})^{2}+\frac{\rho_{i} h_{i}^{3}}{12}\left(\dot{\psi}^{i} \cdot \dot{\psi}^{i}\right)+\rho_{i} h_{i}\left(\dot{v}^{i} \cdot \dot{v}^{i}\right) d \underline{x},
$$

where the "dot product" denotes the usual scalar product on $\mathbf{R}^{2}$.

Define the following $n$ by $n$ matrices:

$$
\begin{array}{ll}
\mathbf{h}=\operatorname{diag}\left(h_{1}, h_{2}, \ldots, h_{n}\right) & \mathbf{D}=\operatorname{diag}\left(D_{1}, D_{2}, \ldots, D_{n}\right) \\
\mathbf{p}=\operatorname{diag}\left(\rho_{1}, \rho_{2}, \ldots, \rho_{n}\right) & \mathbf{G}=\operatorname{diag}\left(G_{1}, G_{2}, \ldots, G_{n}\right)
\end{array}
$$

If $\theta$ and $\xi$ are matrices in $\mathbf{R}^{l m}$, by $\theta \cdot \xi$ we mean the scalar product in $\mathbf{R}^{l m}$. We will also denote

$$
(\theta, \xi)_{\Omega}=\int_{\Omega} \theta \cdot \xi d \underline{x}, \quad(\theta, \xi)_{\Gamma}=\int_{\Gamma} \theta \cdot \xi d \Gamma .
$$

The expressions for the kinetic and potential energy can be rewritten as

$$
\mathcal{K}(t)=c(\dot{v}, \dot{\psi}, \dot{w} ; \dot{v}, \dot{\psi}, \dot{w}) / 2 \quad \mathcal{P}(t)=a(v, \psi, \varphi ; v, \psi, \varphi) / 2
$$

where $c(\cdot ; \cdot)$ and $a(\cdot ; \cdot)$ denote the bilinear forms

$$
\begin{aligned}
c(\psi, v, w ; \hat{\psi}, \hat{v}, \hat{w}) & =((\mathbf{h} \cdot \mathbf{p}) w, \hat{w})_{\Omega}+\left(\left(\mathbf{p h}^{3} / 12\right) \psi, \hat{\psi}\right)_{\Omega}+(\mathbf{h p} v, \hat{v})_{\Omega} \\
a(\psi, v, \varphi ; \hat{\psi}, \hat{v}, \hat{\varphi}) & =a_{0}\left(\mathbf{h}^{3} \psi ; \hat{\psi}\right)+12 a_{0}(\mathbf{h} v ; \hat{v})+a_{1}(\varphi ; \hat{\varphi}) \\
a_{0}(\psi, \hat{\psi}) & =\sum_{i=1}^{n} a_{0}^{i}\left(\psi^{i} ; \hat{\psi}^{i}\right) \\
a_{0}^{i}\left(\psi^{i} ; \hat{\psi}^{i}\right) & =\left(D_{i} \frac{\partial \psi_{1}^{i}}{\partial x_{1}}, \frac{\partial \hat{\psi}_{1}^{i}}{\partial x_{1}}\right)_{\Omega}+\left(D_{i} \frac{\partial \psi_{2}^{i}}{\partial x_{2}}, \frac{\partial \hat{\psi}_{2}^{i}}{\partial x_{2}}\right)_{\Omega} \\
& +\left(\nu_{i} D_{i} \frac{\partial \psi_{2}^{i}}{\partial x_{2}}, \frac{\partial \hat{\psi}_{1}^{i}}{\partial x_{1}}\right)_{\Omega}+\left(\nu_{i} D_{i} \frac{\partial \psi_{1}^{i}}{\partial x_{1}}, \frac{\partial \hat{\psi}_{2}^{i}}{\partial x_{2}}\right)_{\Omega} \\
& +\left(\left(\frac{1-\nu_{i}}{2}\right) D_{i}\left(\frac{\partial \psi_{1}^{i}}{\partial x_{2}}+\frac{\partial \psi_{2}^{i}}{\partial x_{1}}\right),\left(\frac{\partial \hat{\psi}_{1}^{i}}{\partial x_{2}}+\frac{\partial \hat{\psi}_{2}^{i}}{\partial x_{1}}\right)\right)_{\Omega} \\
a_{1}(\varphi ; \hat{\varphi}) & =(\mathbf{G h} \varphi, \hat{\varphi})_{\Omega} .
\end{aligned}
$$

ESAIM: Proc., Vol. 4, 1998, 117-135 
For simplicity, we will assume there are no external forces acting on the plate and no damping is included. To obtain the equations of motion, one first expresses $v, \psi$ and $\varphi$ in terms of the state variables $\{u, w\}$, then forms the Lagrangian from the expressions for the potential and kinetic energy, and finally applies Hamilton's principle.

For example, if the plate is clamped on the boundary we let $\{\hat{u}, \hat{w}\}=$ $\left\{\hat{u}^{0}, \hat{u}^{1}, \ldots, \hat{u}^{n}, \hat{w}\right\}$ denote a test function on $\Omega \times(0, T)$ (with dimensionality matching that of $\{u, w\})$ for which

$$
\begin{gathered}
\{\hat{u}, \hat{w}\}=\left\{\frac{\partial \hat{u}}{\partial n}, \frac{\partial \hat{w}}{\partial n}\right\}=0 \quad \text { on } \Gamma \times(0, T), \\
\left.\{\hat{u}, \hat{w}\}\right|_{t=0}=\left.\frac{\partial}{\partial t}\{\hat{u}, \hat{w}\}\right|_{t=0}=\left.\{\hat{u}, \hat{w}\}\right|_{t=T}=\left.\frac{\partial}{\partial t}\{\hat{u}, \hat{w}\}\right|_{t=T}=0 \quad \text { in } \Omega
\end{gathered}
$$

where $n$ is the outward unit normal to $\Gamma$. An application of Hamilton's principle leads to the equations of motion in weak form:

$$
\int_{0}^{T} c(\dot{\psi}, \dot{v}, \dot{w} ; \dot{\hat{\psi}}, \dot{\hat{v}}, \dot{\hat{w}})-a(\psi, v, \varphi ; \hat{\psi}, \hat{v}, \hat{\varphi}) d t=0
$$

where $\hat{v}, \hat{\psi}$ and $\hat{\varphi}$ are given in terms of $\{\hat{u}, \hat{w}\}$ by (3) (but with hats on $u, v, w, \varphi, \psi)$.

An explicit form of the equations of motion (and corresponding boundary conditions) can be obtained from (9) by a procedure involving an integration by parts in time, and Green's theorem in the spatial variables. The resulting boundary value problem involves a system of $2 n+3$ coupled PDE's whose explicit form is given in Hansen [1]. It is important to note that neither $\psi$ nor $v$ may be used in place of $u$ for a state variable due to the fact that $u$ has $2 n+2$ coordinates while $v$ and $\psi$ each have $2 n$ coordinates. This situation improves, however in the symmetric case.

\subsection{Symmetric multilayer plates}

In the case where the material properties of the multilayer plate are symmetric with respect to the centersheet $\left(x_{3}=0\right.$ in the undeformed configuration), all solutions $\{u, w\}$ of the multilayer plate system decouple into the sum of a symmetric solution $\left\{u_{s}, 0\right\}$ and an antisymmetric solution $\left\{u_{a}, w\right\}$, where $u_{s}$ (resp., $u_{a}$ ) denotes a displacement that is symmetric (resp., antisymmetric) with respect to the centersheet of the multilayer plate. (See [1] for a precise statement about this decoupling.) Since the symmetric part is independent of the transverse displacement, for what concerns bending it is enough to consider only the antisymmetric part. Although this antisymmetric part still satisfies 
the equations of motion (9) (assuming there are $n$ layers), many of these equations become redundant, due to this decoupling. To obtain a minimal set of equations, one needs to make use of the symmetry.

First consider the case in which there are an even number of layers, so that the centersheet of the multilayer plate coincides with the middle interface. Assume that the multilayer plate consists of $2 n$ layers and $2 n+1$ interfaces (this includes the outer faces). Assume that the layers are indexed $i= \pm 1, \pm 2, \ldots, \pm n$ and the interfaces are indexed $i=0, \pm 1, \ldots \pm n$, where the $i=0$ interface represents the centersheet of the multilayer plate, and the $i= \pm 1$ layers are adjacent, and so forth, with the outer layers indexed $\pm n$. Since $u_{i}=-u_{-i}$ (for antisymmetric motions), the motion below the centersheet is exactly opposite that of the top half, and furthermore the potential and kinetic energies of the top and bottom halfs are the same. Consequently it is enough to consider only the energy corresponding to the top half of the composite plate, i.e., those layers indexed from $i=1$ to $i=n$ and those interfaces indexed from $i=0$ to $i=n$. Except for the factor of two, the same Lagrangian is obtained, and consequently (9) is valid for representing the equations of motion, provided that once a solution is obtained, the motions from the bottom half of the plate are determined by antisymmetry. (Note that (9) now applies to the top half of the plate only, as discussed earlier.)

In the case of an odd number of layers, we artificially divide the center layer into two layers with an interface through the centersheet of the middle layer. This effectively creates an even number of layers, reducing the problem to the previously discussed case. Note that we have not changed the problem since no additional degrees of freedom are created in dividing the middle layer. Additional degrees of freedom only contribute to the in-plane solution and do not change the form of the antisymmetric component.

Therefore, when considering the antisymmetric motions, we only need to consider the case of an even number of layers, with the $i=0$ interface representing the center interface. Note that in this case, by the antisymmetry of motions we have that $u_{0} \equiv 0$. As a consequence, only $2 n+1$ state variables are needed (instead of $2 n+3$ ) and hence $\{\psi, w\}$ (or $\{v, w\}$ ) may be used as state variables. If we choose $\{\psi, w\}$, we first express $v$ in terms of $\psi$ (using (3) and that $\left.u^{0}=0\right)$ and then apply the usual integrations by parts to (9).

Before writing down the boundary value problem associated with (9) we first need to develop some notation.

ESAIM: Proc., Vol. 4, 1998, 117-135 


\subsubsection{Notation}

Define

$$
L^{i} \phi=\left\{L_{1}^{i} \phi, L_{2}^{i} \phi\right\}
$$

by

$$
\begin{aligned}
L_{j}^{i} \phi=\frac{\partial}{\partial x_{j}}\left(D_{i} \frac{\partial \phi_{j}}{\partial x_{j}}\right) & +\frac{\partial}{\partial x_{k}}\left(\frac{\left(1-\nu_{i}\right)}{2} D_{i} \frac{\partial \phi_{j}}{\partial x_{k}}\right)+\frac{\partial}{\partial x_{k}}\left(\frac{\left(1-\nu_{i}\right)}{2} D_{i} \frac{\partial \phi_{k}}{\partial x_{j}}\right) \\
& +\frac{\partial}{\partial x_{j}}\left(\nu_{i} D_{i} \frac{\partial \phi_{k}}{\partial x_{k}}\right), \quad(j, k)=(1,2),(2,1) .
\end{aligned}
$$

Also define the boundary operators $\mathcal{B}^{i} \phi=\left\{\mathcal{B}_{1}^{i}\left(\phi_{1}, \phi_{2}\right),\left(\mathcal{B}_{2}^{i}\left(\phi_{1}, \phi_{2}\right)\right\}\right.$ by

$$
\begin{aligned}
& \mathcal{B}_{1}^{i}\left(\phi_{1}, \phi_{2}\right)=D_{i}\left[\left(\frac{\partial \phi_{1}}{\partial x_{1}} n_{1}+\nu_{i} \frac{\partial \phi_{2}}{\partial x_{2}} n_{1}\right)+\left(\frac{1-\nu_{i}}{2}\right)\left(\frac{\partial \phi_{1}}{\partial x_{2}}+\frac{\partial \phi_{2}}{\partial x_{1}}\right) n_{2}\right] \\
& \mathcal{B}_{2}^{i}\left(\phi_{1}, \phi_{2}\right)=D_{i}\left[\left(\frac{\partial \phi_{2}}{\partial x_{2}} n_{2}+\nu_{i} \frac{\partial \phi_{1}}{\partial x_{1}} n_{2}\right)+\left(\frac{1-\nu_{i}}{2}\right)\left(\frac{\partial \phi_{2}}{\partial x_{1}}+\frac{\partial \phi_{1}}{\partial x_{2}}\right) n_{1}\right] .
\end{aligned}
$$

In the above, $\left(n_{1}, n_{2}\right)$ denotes the outward unit normal vector to $\Gamma$. The following Green's formula is valid for all sufficiently smooth $\hat{\phi}, \phi$ :

$$
a_{0}^{i}(\phi, \hat{\phi})=\left(\mathcal{B}^{i} \phi, \hat{\phi}\right)_{\Gamma}-\left(L^{i} \phi, \hat{\phi}\right)_{\Omega}
$$

For $\xi=\left(\xi_{j}^{i}\right)(i=1,2, \ldots, n, j=1,2)$ define the matrices $L \xi$ and $\mathcal{B} \xi$ by

$$
(L \xi)_{i j}=\left(L_{j}^{i} \xi^{i}\right), \quad(\mathcal{B} \xi)_{i j}=\left(\mathcal{B}_{j}^{i} \xi^{i}\right), \quad i=1,2, \ldots, n, j=1,2 .
$$

Let $M$ denote the matrix relating $\psi$ to $v$ :

$$
v=M \psi
$$

\subsubsection{Boundary value problem}

An integration by parts in $t$ of (9) followed by an application of (10) leads to the following:

$$
\begin{array}{ll}
\mathbf{h} \cdot \mathbf{p} \ddot{w}-\operatorname{div} \sum_{i=1}^{n}\left(G_{i} h_{i} \varphi^{i}\right)=0, & \underline{x} \in \Omega, t>0 \\
M^{T} \mathbf{h} \mathbf{p} \ddot{v}-M^{T} \mathbf{h} L v+\mathbf{p h}^{3} \ddot{\psi} / 12-\mathbf{h}^{3} L \psi+\mathbf{G h} \phi=0, & \underline{x} \in \Omega, t>0
\end{array}
$$

where $v=M \psi, \varphi=\psi+\vec{\nabla} w$.

In the case of simply supported boundary conditions (moments and transverse displacement vanish on the boundary) the appropriate boundary conditions are:

$$
w=0, \quad \mathcal{B} \psi=0, \quad \text { on } \Gamma .
$$


For any choice of natural boundary conditions it is possible to show that the resulting boundary value problem is well-posed on the space of finite energy solutions, which is equivalent to $\{\psi, w\}$ belonging the subspace of $\left(H^{1}(\Omega)\right)^{2 n+1}$ determined by the boundary conditions and $\{\dot{\psi}, \dot{w}\}$ belonging to $\left(L^{2}(\Omega)\right)^{2 n+1}$. The reader is referred to [1] for a more detailed discussion of the system (11), and other possible sets of boundary conditions.

\section{$3 \quad$ Laminated plate models}

We describe four models for laminated plates in this section. The first is the initial model based upon the symmetric multilayer model descrbibed in section 2 . The second is obtained from the first as an asymptotic limit, using the assumption that the adhesive layers are thin and compliant. The third is obtained from the second as an asymptotic limit under the assumption that the shear stiffnesses of the plate layers are very large. Our final model is an approximation of the third in which the rotational moments of inertia of the individual plate layers are assumed to be negligible.

The main point is that the low-order model we ultimately obtain may be compared with the initial high-order model and consequently it is possible to bound the range of validity of the low-order model in terms of the high order one.

\subsection{Initial model}

We consider a laminated plate with laminates that are symmetric with respect to the centersheet of the laminated plate. We assume that the laminated plate consists of $2 n$ plate layers and $2 n-1$ adhesive layers. (The case of $2 n+1$ plate layers is similar.) Thus the middle layer is an adhesive layer.

As discussed in the previous subsection, we artificially divide the middle layer into two layers, so that the top and bottom half both consist of $n$ adhesive layers and $n$ adhesive layers. As discussed in Section 2, we only need to consider the top half of the laminated plate. Therefore assume that the bottom (of the top half) is the $i=0$ interface and the top surface is the $i=2 n$ interface. The layers are indexed from $i=1$ to $i=2 n$, with higher layers corresponding to higher indices. Thus adhesive layers are indexed odd and plate layers are indexed even.

To calculate the potential and kinetic energies of the top half, we use the same expressions for the energies of each layer (6), (7), but with $2 n$ instead of $n$ layers. Consequently our initial model for a symmetric laminated plate with $2 n$ plate layers and $2 n$ glue layers is given by (11) but with $n$ replaced by $2 n$.

ESAIM: Proc., Vol. 4, 1998, 117-135 


\subsection{Limit model for thin, compliant adhesive layers}

We now assume that the adhesive layers are thin in comparison to the plate layers and the shear moduli of the adhesive layers are small in comparison to the those of the plate layers. Therefore we treat $\mathbf{h}_{a}$ and $\mathbf{G}_{a}$ as small parameters and would like to obtain a limiting model as these parameters tend to zero. However to retain the potential energy in shear of the adhesive layers we need to retain the ratio $\gamma_{i}:=G_{i} / h_{i}$ fixed for the adhesive layers. We therefore would like to determine the limiting form of the system (11) as $G_{i} \rightarrow 0, h_{i} \rightarrow 0$, with $\gamma_{i}:=G_{i} / h_{i}$ fixed for odd $i$ (adhesive layers).

Before we get to this however we will need some notation to distinguish quantities relating to plate layers from from those of adhesive layers.

Let $P_{a}$ be the projection (a $2 n$ by $n$ matrix) that maps $\left(a_{1}, a_{2}, \ldots, a_{2 n}\right)^{T} \rightarrow$ $\left(a_{1}, a_{3}, \ldots, a_{2 n-1}\right)^{T}$ and define $P_{p}$ to be the corresponding projection that maps to the even-indexed coordinates. For each vector that is indexed by the layers, like $\psi$, for example, we define $\psi_{a}=P_{a} \psi$ and $\psi_{p}=P_{p} \psi$. (We will not need to refer to directional components, so there should be no confusion.) For diagonalmatrix quantities, $\mathbf{h}$, for example, we define $\mathbf{h}_{a}$ to be the $n$ by $n$ diagonal matrix whose diagonal elements are the thicknesses of the adhesive layers (in symbols: $\mathbf{h}_{a}=P_{a} \mathbf{h} P_{a}^{T}$ ). Likewise $\mathbf{h}_{p}$ denotes the diagonal matrix of plate layer thicknesses.

We are interested in finding the limiting form of (11) as

$$
\mathbf{h}_{a} \rightarrow 0, \quad \mathbf{G}_{a} \rightarrow 0 \text { with } \gamma=\mathbf{h}_{a}^{-1} \mathbf{G}_{a} \text { fixed. }
$$

Due the possibility of large shears in the adhesive layers we define a new state variable for these layers:

$$
\delta_{a}=\mathbf{h}_{a} \psi_{a} .
$$

By (3) we see that $\delta_{a}^{i}=u^{i}-u^{i-1}$ ( $i$ odd) and hence represents a differential in displacement of the adhesive layers.

We will wish to use $\left\{w, \psi_{p}, \delta_{a}\right\}$ as state variables. Therefore we need to express the potential and kinetic energies in terms of these quantities. Furthermore, to obtain the equations of motion explicitly, we will need to express $v$ in terms of $\delta_{a}$ and $\psi$. Using that $u^{0} \equiv 0$ (since this is the middle and we are considering only antisymmetric motions) we obtain the following.

$$
\begin{array}{r}
\left(\begin{array}{c}
v_{a} \\
v_{p}
\end{array}\right)=\left(\begin{array}{cc}
(N+I / 2) & N \\
(N+I) & (N+I / 2)
\end{array}\right)\left(\begin{array}{c}
\delta_{a} \\
\mathbf{h}_{p} \psi_{p}
\end{array}\right)=: \\
=:\left(\begin{array}{cc}
A & B \\
C & A
\end{array}\right)\left(\begin{array}{c}
\delta_{a} \\
\mathbf{h}_{p} \psi_{p}
\end{array}\right),
\end{array}
$$


where $N$ denotes the nilpotent matrix with $1^{\prime} s$ everywhere below the main diagonal and 0's everywhere else.

The bilinear forms $c$ and $a$ can be rewritten:

$c(\psi, v, w ; \hat{\psi}, \hat{v}, \hat{w})=\left(\left(\mathbf{h}_{a} \cdot \mathbf{p}_{a}+\mathbf{h}_{p} \cdot \mathbf{p}_{p}\right) w, \hat{w}\right)_{\Omega}+c_{a}\left(\delta_{a}, \psi_{p} ; \hat{\delta}_{a}, \hat{\psi}_{p}\right)+c_{p}\left(\delta_{a}, \psi_{p} ; \hat{\delta}_{a}, \hat{\psi}_{p}\right)$,

$a(\psi, v, \varphi ; \hat{\psi}, \hat{v}, \hat{\varphi})=a_{a}\left(\delta_{a}, \psi_{p} ; \hat{\delta}_{a}, \hat{\psi}_{p}\right)+a_{p}\left(\delta_{a}, \psi_{p} ; \hat{\delta}_{a}, \hat{\psi}_{p}\right)$

where $c_{a}$ and $c_{p}$ are the bilinear forms corresponding to the in-plane kinetic energy of the adhesive layers, and plate layers, respectively, and $a_{a}$ and $a_{p}$ correspond to the strain energies of the adhesive and plate layers, respectively. For example,

$$
\begin{aligned}
& c_{a}\left(\delta_{a}, \psi_{p} ; \hat{\delta}_{a}, \hat{\psi}_{p}\right)=\frac{1}{12}\left(\mathbf{p}_{a} \mathbf{h}_{a} \delta_{a}, \hat{\delta}_{a}\right)_{\Omega}+\left(\mathbf{h}_{a} \mathbf{p}_{a} v_{a}, A \hat{\delta}_{a}+B \mathbf{h}_{p} \hat{\psi}_{p}\right)_{\Omega}, \\
& c_{p}\left(\delta_{a}, \psi_{p} ; \hat{\delta}_{a}, \psi_{p}\right)=\frac{1}{12}\left(\left(\mathbf{p}_{p} \mathbf{h}^{3}\right) \psi_{p}, \hat{\psi}_{p}\right)_{\Omega}+\left(\mathbf{h}_{p} \mathbf{p}_{p} v_{p}, C \hat{\delta}_{a}+A \mathbf{h}_{p} \hat{\psi}_{p}\right)_{\Omega},
\end{aligned}
$$

where $v_{p}$ and $v_{a}$ are defined in terms of the state variables by (14).

Taking the limit in (13) we find that $c_{p}$ and $a_{p}$ are unchanged, while

$$
c_{a} \rightarrow 0, \quad a_{a} \rightarrow\left(\gamma \delta_{a}, \hat{\delta}_{a}\right)_{\Omega} .
$$

We remark that it will be clear how to include the mass of the adhesive layers in the model at a later stage, if this is significant.

The equations of motion can now be calculated in the same way as before. We obtain

$$
\begin{array}{lr}
\mathbf{h}_{p} \cdot \mathbf{p}_{p} \ddot{w}-\operatorname{div} \sum_{i=1}^{n}\left(G_{2 i} h_{2 i} \varphi^{2 i}\right)=0, & \underline{x} \in \Omega, t>0 \\
C^{T}\left(\mathbf{h}_{p} \mathbf{p}_{p} \ddot{v}_{p}-12 \mathbf{h}_{p} L_{p} v_{p}\right)+\gamma \delta_{a}=0, & \underline{x} \in \Omega, t>0 \\
\mathbf{h}_{p} A^{T}\left(\mathbf{h}_{p} \mathbf{p}_{p} \ddot{v}_{p}-12 \mathbf{h}_{p} L_{p} v_{p}\right)+\frac{1}{12} \mathbf{h}_{p}^{3} \mathbf{p}_{p} \ddot{\psi}_{p}-\mathbf{h}_{p}^{3} L_{p} \psi_{p}+\mathbf{G}_{p} \mathbf{h}_{p} \varphi_{p}=0, & \underline{x} \in \Omega, t>0
\end{array}
$$

where $v_{p}$ is given by (14), and $\varphi=\psi+\vec{\nabla} w$, as before.

The simply supported boundary conditions are

$$
w=0, \quad \mathcal{B}_{p} v_{p}=0, \quad \mathcal{B}_{p} \psi_{p}=0 .
$$

For other sets of boundary conditions and comparisons with other plate theories, see $[2])$.

The system of equations (16) is a generalization of the interfacial slip model in Hansen [2], in which only one adhesive layer is present. The "interfacial slip" refers to the variables $\delta_{a}$, which can be viewed as small interfacial slips between the plate layers once the limit (13) has been passed. (Recall that we assume no slip occurs beween plate layers and the adjacent adhesive layers.) Alternatively, $\delta_{a}$ can be viewed as a measure of the shear in the adhesive layers, if one considers those layers to have positive thickness.

An important property is that the limit in (13) is in fact a regular perturbation with respect to the finite energy space. Thus the perturbation does not greatly change the solution, provided that these parameters were sufficiently

ESAIM: Proc., Vol. 4, 1998, 117-135 
small to begin with. (The proof of this will appear in a later paper.) As a consequence of this, the regularity properties, spectral properties, wave speeds, etc., of the the model (16) are nearly the same as that of the original model (11).

\subsection{Limit model for stiff plate layers}

Due to the fact that the adhesive layers have a small shear modulus in comparison to the plate layers, most of the shear motions will occur within the adhesive layers. This suggests that the solutions would not be greatly different if the shear stiffnesses of the plate layers were taken to be infinite.

However, unlike the previous limit, letting the shear stiffness tend to infinity results in a singular perturbation and it is not obvious that limiting solutions (if any) are close to (in some sense) solutions of the original system. Furthermore, it is not obvious how one formally obtains a limiting form of the system (16).

Nevertheless, this problem has been carefully analyzed in Hansen [2] for the case of one adhesive layer and the same basic reasoning applies for this case. One begins by considering a sequence of solutions to the previous system corresponding to increasingly large values of shear stiffness in each layer. The initial data are fixed and such that no energy is stored in shear. By energy estimates one can prove that the sequence of solutions converges in a certain weak sense to a unique limit, which is itself the solution of a certain limiting problem. A detailed discussion of this is impossible here, so we simply indicate the steps one takes to obtain this limiting problem.

Step 1: First, drop the term in $a_{a}$ involving $\mathbf{G}_{p}$. (See (8).) This is due to the fact that in the limit, the amount of energy in shear motions of the plate can be shown to go to zero.

Step 2: Set $\delta_{p}=-\mathbf{h}_{p} \vec{\nabla} w$ in the bilinear forms $a_{a}, a_{p}, c_{p}$. (Recall that $\vec{\nabla} w$ is the matrix with $(\nabla w)^{T}$ in each row.) This corresponds to the fact that in the limit all shear motions are eliminated in the plate layers. Equivalently, the Kirchhoff hypothesis becomes valid for these plate layers.

Before writing down the limiting form of the bilinear forms, note that the only material property remaining from the adhesive layers is $\gamma$. All other material parameters are from plate layers. Therefore we will henceforth omit the subscript $b$ as it is understood. Likewise we will refer to $\delta_{a}$ simply as $\delta$. One finds that

$$
\begin{aligned}
& c \rightarrow(\mathbf{h} \cdot \mathbf{p} w, \hat{w})_{\Omega}+\frac{1}{12}\left(\mathbf{p h}^{3} \vec{\nabla} w, \vec{\nabla} \hat{w}\right)_{\Omega}-\left(\mathbf{h} A^{T} \mathbf{h} \mathbf{p} v_{p}, \vec{\nabla} \hat{w}\right)_{\Omega}+\left(C^{T} \mathbf{h} \mathbf{p} v_{p}, \hat{\delta}\right)_{\Omega} \\
& a \quad \rightarrow a_{0, p}\left(\mathbf{h}^{3} \vec{\nabla} w ; \vec{\nabla} \hat{w}\right)_{\Omega}+12 a_{0, p}\left(\mathbf{h} v_{p} ; C \hat{\delta}-A \mathbf{h} \vec{\nabla} \hat{w}\right)+(\gamma \delta, \hat{\delta})_{\Omega} .
\end{aligned}
$$

Step 3: Denote by $\overrightarrow{1}$ the column vector consisting of $n 1$ 's, so that $\vec{\nabla} w=$ 
$\overrightarrow{1} \nabla w$. An integration by parts in $t$ of (9) followed by an application of the Green's formula (10) leads to the following:

$\mathbf{h} \cdot \mathbf{p} \ddot{w}-\operatorname{div} \overrightarrow{1}^{T} \frac{\mathbf{p h}^{3}}{12} \overrightarrow{1} \nabla \ddot{w}+\operatorname{div} \overrightarrow{1}^{T} \mathbf{h} A^{T} \mathbf{h} \mathbf{p} \ddot{v}_{p}+\operatorname{div} \overrightarrow{1}^{T} L \mathbf{h}^{3} \overrightarrow{1} \nabla w-12 \operatorname{div} \overrightarrow{1}^{T} \mathbf{h} A^{T} L \mathbf{h} v_{p}=0$

$$
C^{T} \mathbf{h p} \ddot{v}_{p}-12 C^{T} L \mathbf{h} v_{p}+\gamma \delta=0 .
$$

where $v_{p}=C \delta-A \mathbf{h} \overrightarrow{1} \nabla w$.

In the case of simply supported boundary conditions with no applied moments acting on the adhesives on $\Gamma$ (the boundary), the boundary conditions are

$$
w=0, \quad \mathcal{B} v_{p}=0, \quad \overrightarrow{1}^{T} \mathbf{h}^{3} \mathcal{B} \overrightarrow{1} \nabla w \cdot n=0, \quad \text { on } \Gamma .
$$

In the case of a single adhesive layer, the system (17), (18) reduces to one obtained by similar means in Hansen [2]. System (17), (18) is also related to the Kirchhoff plate model in some limiting cases we discuss below.

First let us examine the limiting behavior as the adhesive stiffness of each adhesive layer tends to $\infty$.

\subsubsection{Limit as $\gamma \rightarrow \infty$}

As $\gamma \rightarrow \infty$ the shear motions in the adhesive layers become increasingly penalized and in the limit such motions would imply infinite energy. This suggest simply putting $\delta=0$ in (17) to obtain the limiting system. However, again, this limit results in a singular perturbation and consequently it is not obvious that limiting solutions (if any) are close in any sense to those of the initial system. Nevertheless, by an analysis similar to one in Hansen [2] it can be shown that one obtains convergence of solutions in a certain weak star topology.

Putting $\delta=0$ in (17) and simplifying, we obtain

$$
m \ddot{w}-\alpha_{\infty} \triangle \ddot{w}+K_{\infty} \triangle^{2} w=0
$$

where

$$
m=\sum_{i=1}^{n} h_{i} \rho_{i}, \quad \alpha_{\infty}=\overrightarrow{1}^{T}\left(\frac{\mathbf{p h}}{12}+\mathbf{h} A^{T} \mathbf{h} \mathbf{p} A \mathbf{h}\right) \overrightarrow{1}, \quad K_{\infty}=\overrightarrow{1}^{T}\left(\mathbf{D} \mathbf{h}^{3}+12 \mathbf{h} A^{T} \mathbf{h D} A \mathbf{h}\right) \overrightarrow{1} .
$$

In the case where $\rho_{i}=\rho_{0}$, all $i$, (same density for each beam layer) $m=\rho_{0} \tau / 2$, where $\tau=2 \sum_{i=1}^{n} h_{i}$ is the total thickness of the beam, and $\alpha_{\infty}=(1 / 2) \rho_{0} \tau^{3} / 12$ is the half of the mass moment of inertia of the beam. If $D_{i}=D_{0}$, all $i$, then $K=D_{0} \tau^{3} / 2$ is half the stiffness of the beam. (Our factor of $1 / 2$ appearing in all terms is due to calculating the energy of the upper half of the plate.)

ESAIM: Proc., Vol. 4, 1998, 117-135 
Thus as the adhesive becomes increasingly stiff, if the densities and Young's moduli of each layer are the same, the system (17), (18) reduces to the usual Kirchhoff plate. In more general cases where the densities or Youngs' moduli of the layers are not constant the expressions for $m, \alpha_{\infty}$ and $K_{\infty}$ give the effective constants for (half of) the longitudinal inertia, rotational inertia and stiffness, respectively, for a layered plate in which the layers are perfectly bonded to one another.

\subsubsection{Limit as $\gamma \rightarrow 0$}

Let us now consider the limiting behavior as $\gamma \rightarrow 0$. Physically, when there is no (or very little) adhesive bonding the layers, the moment of inertias of the layers are no longer coupled and the stiffness the entire beam should reduce to the sum of the stiffnesses of each layer. Indeed this is what one finds.

If $\gamma=0$ there is no resistance to shear in the adhesive layers, and hence the term due to shear potential energy in (8) will vanish. In this case (18) reduces to a hyperbolic PDE with (assuming simply supported boundary conditions) homogeneous boundary conditions. Assuming initial conditions such that $v$ and $\dot{v}$ are zero at time zero, the unique solution of this system is the zero function. Putting $v_{p}=0$ into (17) results in

$$
\frac{1}{2}\left(m_{0} \ddot{w}-\alpha_{0} \triangle \ddot{w}+K_{0} \triangle^{2} w\right)=0
$$

where

$$
m_{0}=\sum_{i=1}^{n} h_{i} \rho_{i}, \quad \alpha_{0}=\frac{1}{12} \sum_{i=1}^{n} \rho_{i} h_{i}^{3}, \quad K=\sum_{i=1}^{n} D_{i} h_{i}^{3} .
$$

Thus term $m_{0}$ remains the same as in (20), the moment of inertia term $\alpha_{0}$ reduces to the sum of the moments of inertia of each layer, while $K_{0}$ becomes the linear sum of the stiffnesses of each layer.

In the case where $\rho_{i}=\rho_{0}$, all $i$, (same density of each beam layer) $m=\rho_{0} \tau$, where $\tau=2 \sum_{i=1}^{n} h_{i}$ is the total thickness of the beam, and $\alpha_{0}=\rho_{0} \tau^{3} / 12$ is the mass moment of inertia of the beam. If $D_{i}=D_{0}$, all $i$, then $K=D_{0} \tau^{3}$ is the stiffness of the plate.

\subsection{Long wave approximation}

If the plate layers are very thin, or if the frequencies of vibration are not too high, then the inertial terms in (18) are negligible compared to the stiffness terms. Neglecting those inertial terms in (18) gives

$\mathbf{h} \cdot \mathbf{p} \ddot{w}-\frac{1}{12} \operatorname{div} \overrightarrow{1}^{T} \mathbf{p h}^{3} \overrightarrow{1} \nabla \ddot{w}+\operatorname{div} \overrightarrow{1}^{T} \mathbf{h} A^{T} \mathbf{h} \mathbf{p} \ddot{v}_{p}+\operatorname{div} \overrightarrow{1}^{T} L \mathbf{h}^{3} \overrightarrow{1} \nabla w-12 \operatorname{div} \overrightarrow{1}^{T} \mathbf{h} A^{T} L \mathbf{h} v_{p}=0$, 


$$
-12 C^{T} L \mathbf{h} v_{p}+\gamma \delta=0
$$

where $v_{p}=C \delta-A \mathbf{h} \overrightarrow{1} \nabla w$.

We have retained the rotational inertia term in (23) since this reflects the net rotational energy of the entire laminated plate.

With the inertial terms gone, (24) is an eliptic system in which $\delta$ may be determined in terms of $\vec{\nabla} w$. Once this is substituted back in (23), a scalar equation results.

Let us define $R$ by $R \phi=\mathbf{h} \mathbf{p} \ddot{\phi}-12 L \mathbf{h} \phi$ and $R_{0}$ by $R_{0} \phi=-12 L \mathbf{h} \phi$. We have

$$
v_{p}=C \delta-A \mathbf{h} \overrightarrow{1} \nabla w=\left(-R_{0}\right)^{-1}\left(C^{T}\right)^{-1} \gamma \delta .
$$

Hence (23), (24) can be written

$$
\mathbf{h} \cdot \mathbf{p} \ddot{w}-\operatorname{div} \overrightarrow{1}^{T} \mathbf{h}\left[\frac{1}{12} R+A^{T} R P A\right] \mathbf{h} \overrightarrow{1} \nabla w=0
$$

where

$$
P=\left(C^{T} R_{0}+\gamma C^{-1}\right)^{-1} \gamma C^{-1} .
$$

Therefore the plate system becomes

$$
m \ddot{w}-\alpha \triangle \ddot{w}+K \triangle^{2} w=0,
$$

where

$$
\begin{aligned}
m & =\mathbf{p} \cdot \mathbf{h}=\sum_{i=1}^{n} \rho_{i} h_{i}=\rho_{0} h / 2 \\
\alpha & =\overrightarrow{1}^{T} \frac{1}{12} \mathbf{p h}^{3} \overrightarrow{1}+\overrightarrow{1}^{T} \mathbf{h} A^{T} \mathbf{h} \mathbf{p} P A \mathbf{h} \overrightarrow{1} \\
K & =\overrightarrow{1}^{T} \mathbf{D} h^{3} \overrightarrow{1}+12 \overrightarrow{1}^{T} \mathbf{h} A^{T} \mathbf{h D} P A \mathbf{h} \overrightarrow{1} .
\end{aligned}
$$

Thus, the system reduces to one which resembles a standard Kirchhoff plate, although the coefficients $\alpha$ and $K$ contain the operator $P$.

Often in engineering applications the coefficients in a beam or plate are said to be "frequency dependent", which means simply that they adjust the values depending upon what a relevant mean frequency might be. One can easily determine the predicted behavior of the parameters $\alpha$ and $K$ as a function of frequency by a spectral analysis.

Let us examine the operator $P$ more closely. Suppose $\left(\lambda_{k}\right), k=1,2, \ldots$ are the eigenvalues of $\triangle$, arranged in nondecreasing order and let $\phi_{k}$ denote the corresponding eigenfunctions. In one dimension, $\lambda_{k}=-k^{2} \pi^{2} /\left(L^{2}\right)$ and 
$\phi_{k}=\sin (k \pi x / L)$, where $L$ is the length of the interval. If $\left|\lambda_{k}\right|$ is small (in comparison to $\gamma$ ) then

$$
P \phi_{k}=-\left(C^{T} \lambda_{k}+\gamma C^{-1}\right)^{-1} \gamma C^{-1} \phi_{k} \approx I \phi_{k} \quad \text { (low frequency approximation). }
$$

That is, at a low enough frequency $P \approx I$. On the other hand, as $\lambda_{k}$ gets large, $\triangle I$ must dominate and

$$
P \rightarrow 0 \quad \text { (high frequency approximation). }
$$

It is also easy to see that the $P$ is a monotonic function of the frequency.

Thus, we can make the following observations.

At very low frequencies, the rotational inertia parameter $\alpha$ and the stiffness parameter $K$ tend to the values in (20), which correspond to the case in which the adhesive is infinitely strong. Of course, the degree of similarity is limited, due to the fact that the lowest eigenvalue may still be quite large.

As the frequency increases, $P$ tends to zero, and hence $\alpha$ and $K$ tend to the values in (22), which is simply the linear sum of the rotational inertias and stiffnesses of each layer. Thus at higher frequencies the laminated plate (23), (24) becomes increasingly flimsy in comparison to a similar plate that neglects the effect of the adhesive.

\section{Acknowledgement}

Research for this project was supported in part by the National Science Foundation under Grant DMS9623144.

\section{References}

[1] S. W. Hansen. A dynamical model form multilayered plates with independent shear deformations. Q. Appl. Math., (to appear).

[2] S. W. Hansen. A model for a two-layered plate with interfacial slip. In F. Kappel W. Desch and K. Kunisch, editors, Estimation and Control of Distributed Parameter Systems: Nonlinear Phenomena, volume 118, pages 143-170, Basel, 1994. Birkhauser-Verlag.

[3] D. J. Mead. A comparison of some equations for the flexural vibration of damped sandwich beams. J. Sound Vib., pages 363-377, 1982.

[4] R.D. Mindlin. Influence of rotary inertia and shear on flexural motions of isotropic elastic plates. J. Appl. Mech., 18:31-38, 1951. 
[5] E. Reissner. The effect of transverse shear deformations on the bending of elastic plates. J. Appl. Mech., 12:A69-A77, 1945.

[6] C. T. Sun and Y. P. Lu. Vibration Damping for Structural Elements. Prentice Hall, Englewood Cliffs, New Jersey, 1995.

[7] M.-J. Yan and E. H. Dowell. Governing equations for vibrating constrainedlayer damping sandwich plates and beams. J. Appl. Mech., pages 1041-1046, 1972.

ESAIM: Proc., Vol. 4, 1998, 117-135 